NYU-TH-96/02/02

February 1996

\title{
Consistency Condition for the Pinch Technique Self-Energies at Two Loops
}

\author{
Kostas Philippides and Alberto Sirlin \\ New York University, Department of Physics \\ Andre and Bella Meyer Hall of Physics, 4 Washington Place \\ New York, NY 10003, USA.
}

\begin{abstract}
A simple and testable necessary condition for the gauge independence of the Pinch Technique self-energies at two loops is discussed. It is then shown that, in the case of the $Z$ and $W$ self-energies, the condition is indeed satisfied by the Papavassiliou-Pilaftsis formulation.
\end{abstract}


The Pinch Technique (PT) is a convenient algorithm that automatically rearranges S-matrix elements of gauge theories into modified, gauge-independent self-energies, vertex, and box diagrams. In turn, the new corrections exhibit very desirable theoretical properties. For these reasons, the PT has been frequently employed in recent discussions of QCD and Electroweak Physics [1]. A temporary drawback is that the approach has been fully developed only at the one-loop level. Very recently, however, Papavassiliou and Pilaftsis (P-P) proposed a method to construct PT self energies at higher orders [2].

Calling $\widehat{\Pi}$ and $\Pi$ the $\mathrm{PT}$ and $R_{\xi}$ transverse self-energies, respectively, and focusing on the $Z$ case, one has

$$
\widehat{\Pi}^{Z Z}(s)=\Pi^{Z Z}(s)+\left(\Pi^{Z Z}(s)\right)^{P},
$$

where the "pinch part" $\left(\Pi^{Z Z}(s)\right)^{P}$ has the structure

$$
\left(\Pi^{Z Z}(s)\right)^{P}=c_{1}\left(s-M_{0}^{2}\right) V^{P}(s)+c_{2}\left(s-M_{0}^{2}\right)^{2} B^{P}(s)-R^{Z Z}(s) .
$$

In Eq.(目) the bare mass $M_{0}$ is assumed to be defined in a gauge invariant manner, tadpole contributions are included in $\Pi^{Z Z}(s), V^{P}(s)$ and $B^{P}(s)$ are the pinch parts from vertex and box diagrams, respectively, and $R^{Z Z}(s)$ is a residual amplitude of $\mathcal{O}\left(g^{4}\right)$ proposed in Ref. [2]. It is discussed in detail later on at the $\mathcal{O}\left(g^{4}\right)$ level. Because of the limited knowledge currently available concerning multi-loop amplitudes in gauge theories, a general proof that $\widehat{\Pi}^{Z Z}(s)$ is gauge invariant in higher orders is not presently available. One of the aims of this report is to note that by judiciously restricting the domain of $s$ to lie in the neighborhood of $\bar{s}$, the complex-valued position of the propagator's pole, one can obtain an expression for which the gauge independence can be tested on the basis of current knowledge. Specifically, we consider the neighborhood $|s-\bar{s}| \leq \mathcal{O}\left(g^{2}|\bar{s}|\right)$, which roughly includes the resonance region. Recalling that $\bar{s}-M_{0}^{2}=\mathcal{O}\left(g^{2}\right)$, through $\mathcal{O}\left(g^{4}\right)$ Eqs.(10,2) become

$$
\widehat{\Pi}^{Z Z}(s)=\Pi_{1}^{Z Z}(s)+c_{1}\left(s-M_{0}^{2}\right) V_{1}^{P}(s)+\Pi_{2}^{Z Z}(\bar{s})-R_{2}^{Z Z}(\bar{s})+\mathcal{O}\left(g^{6}\right),
$$

where the indices $i=1,2$ denote $\mathcal{O}\left(g^{2}\right)$ and $\mathcal{O}\left(g^{4}\right)$ contributions. Through $\mathcal{O}\left(g^{4}\right)$ the first two terms in the r.h.s. of Eq.(3) equal the one-loop PT self-energy $\widehat{\Pi}_{1}^{Z Z}(s)$, which is 
$\xi$-independent . Its explicit expression [3] is :

$$
\widehat{\Pi}_{1}^{Z Z}(s)=\left.\Pi_{1}^{Z Z}(s)\right|_{\xi_{i}=1}-4 g^{2} c_{w}^{2}\left(s-M_{0}^{2}\right) I_{W W}(s),
$$

where $c_{w}^{2}$ is an abbreviation for $\cos \theta_{w}^{2}, \xi_{i}(i=W, Z, \gamma)$ are the $R_{\xi}$ gauge parameters and

$$
I_{i j}\left(q^{2}\right)=i \mu^{4-n} \int \frac{d^{n} k}{(2 \pi)^{n}} \frac{1}{\left(k^{2}-M_{i}^{2}\right)\left[(k+q)^{2}-M_{j}^{2}\right]} .
$$

On the other hand, $\Pi_{2}^{Z Z}(\bar{s})$ in Eq.(四) is expected to be gauge dependent. Although this amplitude is not fully known, its gauge-dependent part can be isolated by a simple argument. Recalling that the pole position $\bar{s}=M_{0}^{2}+\Pi^{Z Z}(\bar{s})+\left[\Pi^{\gamma Z}(\bar{s})\right]^{2} /\left[\bar{s}-\Pi^{\gamma \gamma}(\bar{s})\right]$ is gauge invariant, through $\mathcal{O}\left(g^{4}\right)$ we have

$$
\Pi_{2}^{Z Z}(\bar{s})=\bar{s}-M_{0}^{2}-\widehat{\Pi}_{1}^{Z Z}(\bar{s})+c_{1}\left(\bar{s}-M_{0}^{2}\right) V_{1}^{P}(\bar{s})-\left[\Pi_{1}^{\gamma Z}(\bar{s})\right]^{2} / \bar{s},
$$

where $\widehat{\Pi}_{1}^{Z Z}(\bar{s})$ is defined in Eq.(四). The amplitude $c_{1} V_{1}^{P}(s)$ can be gleaned from Eqs.(12b,16d) of Ref.[3] :

$$
c_{1} V_{1}^{P}(s)=-4 g^{2} c_{w}^{2}\left[I_{W W}(s)+\left(\xi_{W}-1\right) v_{W}(s) / 2\right],
$$

where $v_{W}(s)$ is a $\xi_{i}$-dependent function defined in Eqs.(2-6) of Ref. [4]. Recalling $\bar{s}-M_{0}^{2}=$ $\left.\Pi_{1}^{Z Z}(\bar{s})\right|_{\xi_{i}=1}+\mathcal{O}\left(g^{4}\right)$, we have

$$
c_{1}\left(\bar{s}-M_{0}^{2}\right) V_{1}^{P}(\bar{s})=-\left.4 g^{2} c_{w}^{2} \Pi_{1}^{Z Z}(\bar{s})\right|_{\xi_{i}=1}\left[I_{W W}(\bar{s})+\left(\xi_{W}-1\right) v_{W}(\bar{s}) / 2\right] .
$$

On the other hand, using Eq.(8) of Ref. [4], one finds for general $\xi_{i}$

$$
\begin{aligned}
\frac{\left[\Pi_{1}^{\gamma Z}(\bar{s})\right]^{2}}{\bar{s}}= & {\left[\left.\Pi_{1}^{\gamma Z}(\bar{s})\right|_{\xi_{i}=1}+g^{2} s_{w} c_{w}\left(\xi_{W}-1\right) \bar{s} v_{W}(\bar{s})\right]^{2} / \bar{s}+\mathcal{O}\left(g^{6}\right) } \\
= & {\left[\left.\Pi_{1}^{\gamma Z}(\bar{s})\right|_{\xi_{i}=1}\right]^{2} / \bar{s}+\left.2 g^{2} s_{w} c_{w}\left(\xi_{W}-1\right) v_{W}(\bar{s}) \Pi_{1}^{\gamma Z}(\bar{s})\right|_{\xi_{i}=1} } \\
& +g^{4} s_{w}^{2} c_{w}^{2}\left(\xi_{W}-1\right)^{2} \bar{s} v_{W}^{2}(\bar{s})+\mathcal{O}\left(g^{6}\right) .
\end{aligned}
$$

Combining Eqs.(6.88.9) we obtain

$$
\begin{aligned}
\Pi_{2}^{Z Z}(\bar{s})= & \left.\Pi_{2}^{Z Z}(\bar{s})\right|_{\xi_{i}=1}-2 g^{2}\left(\xi_{W}-1\right) v_{W}(\bar{s})\left[\left.c_{w}^{2} \Pi_{1}^{Z Z}(\bar{s})\right|_{\xi_{i}=1}+\left.c_{w} s_{w} \Pi_{1}^{\gamma Z}(\bar{s})\right|_{\xi_{i}=1}\right] \\
& -g^{4} s_{w}^{2} c_{w}^{2}\left(\xi_{W}-1\right)^{2} \bar{s} v_{W}^{2}(\bar{s}) .
\end{aligned}
$$

The terms proportional to $\xi_{W}-1$ and $\left(\xi_{W}-1\right)^{2}$ in Eq.(10) represent the $\xi_{i}$-dependent parts of $\Pi_{2}^{Z Z}(\bar{s})$. It follows that, if the residual contributions $R_{2}^{Z Z}(\bar{s})$ are not included, 
Eq.(3) is gauge-dependent in $\mathcal{O}\left(g^{4}\right)$. Next we evaluate $R_{2}^{Z Z}(\bar{s})$. Following the P-P method [2], $R_{2}$ is the contribution that must be added to the chain of $R_{\xi}$ transverse one-loop selfenergies and corresponding pinch parts (Fig.1(b-d)), in order to convert it into the chain of one-loop PT transverse self-energies (Fig.1a). The explicit construction of $R_{2}^{Z Z}(\bar{s})$ in the $\xi_{i}=1$ gauges has been given in Ref. [5]. We must now generalize this procedure to a general gauge. The chain of one-loop PT self-energies is by definition $\xi$-independent and gives a contribution proportional to

$$
\left[\left.\Pi_{1}^{Z Z}(s)\right|_{\xi_{i}=1}-\left(s-M_{0}^{2}\right) 4 g^{2} c_{w}^{2} I_{W W}\right]^{2} /\left(s-M_{0}^{2}\right)+\left[\left.\Pi_{1}^{\gamma Z}(s)\right|_{\xi_{i}=1}-\left(2 s-M_{0}^{2}\right) 2 g^{2} s_{w} c_{w} I_{W W}\right]^{2} / s,
$$

where we have employed Eqs.(16d,16b) of Ref.[3]. On the other hand, using the results of Refs. [3], [4] and neglecting $\mathcal{O}\left(g^{6}\right)$, one finds that for $s=\bar{s}$ and general $\xi_{i}$ the chain of $R_{\xi}$ one-loop self-energies and pinch parts contributes

$$
\begin{aligned}
& {\left[\left.\Pi_{1}^{Z Z}(\bar{s})\right|_{\xi_{i}=1}+\left(\bar{s}-M_{0}^{2}\right) 2 g^{2} c_{w}^{2}\left(\xi_{W}-1\right) v_{W}(\bar{s})\right]^{2} /\left(\bar{s}-M_{0}^{2}\right)} \\
& +\left[\left.\Pi_{1}^{\gamma Z}(s)\right|_{\xi_{i}=1}+\bar{s} g^{2} s_{w} c_{w}\left(\xi_{W}-1\right) v_{W}(\bar{s})\right]^{2} / \bar{s} \\
& -\left.4 g^{2} c_{w}^{2} \Pi_{1}^{Z Z}(\bar{s})\right|_{\xi_{i}=1}\left[I_{W W}(\bar{s})+\left(\xi_{W}-1\right) v_{W}(\bar{s}) / 2\right]
\end{aligned}
$$

The two first terms arise from the self-energy contributions in a general $R_{\xi}$ gauge [4] through $\mathcal{O}\left(g^{4}\right)$, while the third involves the contribution of self-energies and pinch parts (Fig.1(b-d)) in the same approximation. Setting $s=\bar{s}=M_{0}^{2}+\Pi_{1}^{Z Z}(\bar{s})+\ldots$ in Eqs. (11).12) and subtracting the two expressions we obtain

$$
\begin{gathered}
R_{2}^{Z Z}(\bar{s})=-\left.4 g^{2}\left[c_{w}^{2} \Pi_{1}^{Z Z}(\bar{s})+s_{w} c_{w} \Pi_{1}^{\gamma Z}(\bar{s})\right]\right|_{\xi_{i}=1}\left[I_{W W}(\bar{s})+\left(\xi_{W}-1\right) v_{W}(\bar{s}) / 2\right] \\
+g^{4} s_{w}^{2} c_{w}^{2} \bar{s}\left[4 I_{W W}^{2}(\bar{s})-\left(\xi_{W}-1\right)^{2} v_{W}^{2}(\bar{s})\right]+\mathcal{O}\left(g^{6}\right) .
\end{gathered}
$$

Comparing Eq.(10) with Eq.(13) we see that the $\xi_{W}$-dependent contributions cancel in the combination $\Pi_{2}^{Z Z}(\bar{s})-R_{2}^{Z Z}(\bar{s})$. Thus, Eq.(3) is indeed gauge-independent through $\mathcal{O}\left(g^{4}\right)$ if $R_{2}^{Z Z}(\bar{s})$ is evaluated according to the P-P method. In the neighborhood $|s-\bar{s}| \leq$ $\mathcal{O}\left(g^{2}|\bar{s}|\right)$, Eq.(3i) becomes

$$
\begin{aligned}
& \widehat{\Pi}^{Z Z}(s)= \begin{array}{c}
\left.\Pi_{1}^{Z Z}(s)\right|_{\xi_{i}=1}+\left.\Pi_{2}^{Z Z}(\bar{s})\right|_{\xi_{i}=1}-4 g^{2} c_{w}^{2}(s-\bar{s}) I_{W W}(\bar{s}) \\
+
\end{array} \\
&+\left.4 g^{2} s_{w} c_{w} \Pi_{1}^{\gamma Z}(\bar{s})\right|_{\xi_{i}=1} I_{W W}(\bar{s})-4 g^{4} s_{w}^{2} c_{w}^{2} \bar{s} I_{W W}^{2}(\bar{s})+\mathcal{O}\left(g^{6}\right) .
\end{aligned}
$$


For $s=\bar{s}$, Eq.(14) reduces to Eq.(3.16) of Ref.[5]. Using Eq.(14) one finds that in the PT approach the denominator in the $Z$ propagator can be written as

$$
s-M_{0}^{2}-\widehat{\Pi}^{Z Z}(s)-\left[\widehat{\Pi}^{\gamma Z}(s)\right]^{2} / s=(s-\bar{s})\left[1-\left.\frac{d}{d s} \widehat{\Pi}_{1}^{Z Z}(s)\right|_{s=\bar{s}}\right]+\mathcal{O}\left(g^{6}\right),
$$

where it is understood that $|s-\bar{s}| \leq \mathcal{O}\left(g^{2}|\bar{s}|\right)$. In order to derive Eq.(15), it is convenient to add and subtract $\bar{s}$ in the l.h.s., employ $\bar{s}-M_{0}^{2}=\Pi^{Z Z}(\bar{s})+\left(\Pi^{\gamma Z}(\bar{s})\right)^{2} / \bar{s}+\ldots$, and recall the expression for $\widehat{\Pi}^{\gamma Z}$ given in Eq.(16b) of Ref[3]. Eq.(15) explicitly shows two important properties: 1$)$ as it involves the PT self-energy $\widehat{\Pi}_{1}^{Z Z}(s)$, it is manifestedly $\xi_{i}$-independent 2) the zero of Eq.(15) occurs at $s=\bar{s}$, so that the pole position is not displaced. For the $Z$ case, the latter property was already derived in the particular case of the $\xi_{i}=1$ gauges [5]. As explained in Refs. [5], [6], using the scaling approximation for $\mathcal{I} m \widehat{\Pi}_{1}^{Z Z}(s)$ one can transform Eq.(15) into the characteristic $s$-dependent Breit-Wigner resonance employed in the LEP analysis, so that the connection with the LEP observables becomes explicit.

One can readily carry out the same analysis for the $W$ self-energy . In this case there are no mixing complications but the $R_{\xi}$ gauge dependence is governed by three parameters $\xi_{i}(i=W, Z, \gamma)$. One finds

$$
\begin{gathered}
\Pi_{2}^{W W}(\bar{s})=\left.\Pi_{2}^{W W}(\bar{s})\right|_{\xi_{i}=1}-\left.g^{2} \Pi_{1}^{W W}(\bar{s})\right|_{\xi_{i}=1} F\left(\xi_{i}, \bar{s}\right)+\mathcal{O}\left(g^{6}\right), \\
F\left(\xi_{i}, \bar{s}\right)=c_{w}^{2}\left[\left(\xi_{W}-1\right) v_{W Z}(\bar{s})+(W \leftrightarrow Z)\right]+s_{w}^{2}\left[\left(\xi_{W}-1\right) v_{W \gamma}(\bar{s})+(W \leftrightarrow \gamma)\right],
\end{gathered}
$$

where $(i \leftrightarrow j)$ is obtained from the preceding term by interchanging the indices in $\left(\xi_{i}-\right.$ 1) $v_{i j}(\bar{s})$. The gauge dependence is contained in $F\left(\xi_{i}, \bar{s}\right)$. Following the P-P method we obtain

$$
R_{2}^{W W}(\bar{s})=-\left.4 g^{2} \Pi_{1}^{W W}(\bar{s})\right|_{\xi_{i}=1}\left[c_{w}^{2} I_{Z W}(\bar{s})+s_{w}^{2} I_{\gamma W}(\bar{s})\right]-\left.g^{2} \Pi_{1}^{W W}(\bar{s})\right|_{\xi_{i}=1} F\left(\xi_{i}, \bar{s}\right) .
$$

Again $\Pi_{2}^{W W}(\bar{s})-R_{2}^{W W}(\bar{s})$ is $\xi_{i}$-independent and in the interval $|s-\bar{s}| \leq \mathcal{O}\left(g^{2}|\bar{s}|\right)$ we find

$$
\widehat{\Pi}^{W W}(s)=\left.\Pi_{1}^{W W}(s)\right|_{\xi_{i}=1}+\left.\Pi_{2}^{W W}(\bar{s})\right|_{\xi_{i}=1}-4 g^{2}(s-\bar{s})\left[c_{w}^{2} I_{Z W}(\bar{s})+s_{w}^{2} I_{\gamma W}(\bar{s})\right]+\mathcal{O}\left(g^{6}\right) .
$$

Alternatively, the $\xi_{i}$-independence of $\widehat{\Pi}_{2}^{W W}(\bar{s})$ can be derived by directly evaluating $\widehat{\Pi}_{1}^{W W}(\bar{s})+\widehat{\Pi}_{2}^{W W}(\bar{s})-\Pi_{1}^{W W}(\bar{s})-\Pi_{2}^{W W}(\bar{s})$ in a general $\xi_{i}$ gauge [2]]. Using Eq.(19]) the 
propagator's denominator becomes

$$
s-\left(M_{0}^{W}\right)^{2}-\widehat{\Pi}^{W W}(s)=(s-\bar{s})\left[1-\left.\frac{d}{d s} \widehat{\Pi}_{1}^{W W}(s)\right|_{s=\bar{s}}\right]+\mathcal{O}\left(g^{6}\right),
$$

in analogy with the $Z$ case.

In summary, by restricting $s$ to the neighborhood $|s-\bar{s}| \leq \mathcal{O}\left(g^{2}|\bar{s}|\right)$ of the propagator's pole one can test the gauge dependence of the $Z Z$ and $W W$ self-energies through $\mathcal{O}\left(g^{4}\right)$. In both cases we find that the P-P method leads to gauge independent amplitudes. Because of our restriction to the resonance region, and our neglect of $\mathcal{O}\left(g^{6}\right)$ terms, this test amounts to a necessary rather than a sufficient condition. On the other hand, it is important to emphasize that this domain is of special physical significance.

This work was supported in part by the National Science Foundation under grant No. PHY-9313781.

\section{Figure Caption}

Chain of one-loop transverse PT self-energies through $\mathcal{O}\left(g^{4}\right)$ and a class of related pinch parts.

\section{References}

[1] J.M. Cornwall, Phys. Rev. D26 (1982) 1453; J.M. Cornwall and J. Papavassiliou, Phys. Rev. D40 (1989) 3474; J. Papavassiliou, Phys. Rev. D41 (1990) 3179; Phys. Rev. D47 (1993) 4728; Phys. Rev. D50 (1994) 5958 ; G. Degrassi, B. Kniehl, and A. Sirlin, Phys. Rev. D48 (1993) R3963; J. Papavassiliou and K. Philippides Phys. Rev. D48 (1993) 4255; J. Papavassiliou and C. Parrinello, Phys. Rev. D50 (1994) 3059; K.Hagiwara, D.Haidt, C.S.Kim, and S.Matsumoto, Z. Phys. C64 (1994) 559; J. Papavassiliou and A. Sirlin, Phys. Rev. D50 (1994) 5951; N.J. Watson, Phys. Lett B349 (1995) 155; J. Papavassiliou and K. Philippides, Phys. Rev. D53 (1995) 2355; K. Sasaki, hep-ph/9510311 to be published in Phys. Lett B; hep-ph/9512434.

[2] J. Papavassiliou and A. Pilaftsis, Phys. Rev. Let. 75 (1995) 3060; Phys. Rev. D53 2128 (1996). 
[3] G. Degrassi and A. Sirlin, Phys. Rev. D46 (1992) 3104.

[4] G. Degrassi and A. Sirlin, Nucl. Phys. B383 (1992) 73.

[5] K. Philippides and A. Sirlin, Phys. Lett. B367 (1996) 377.

[6] A. Sirlin, Phys. Rev. Lett. 67 (1991) 2127; Phys. Lett. B267 (1991) 240. 


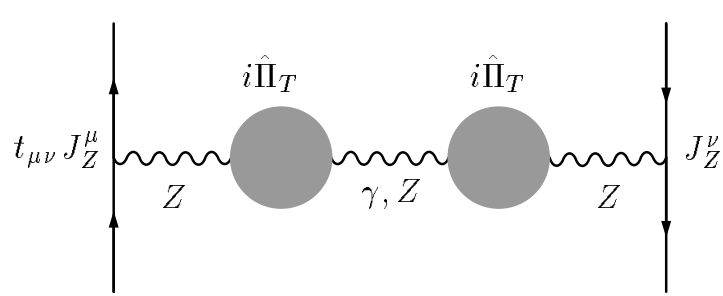

(a)

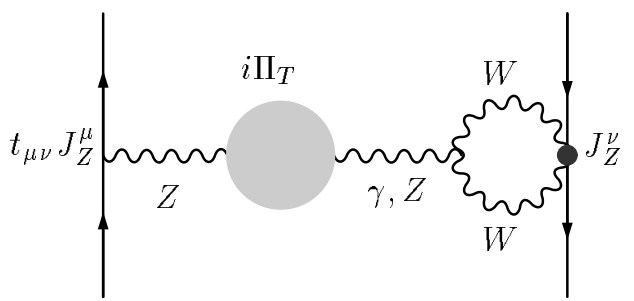

(b)

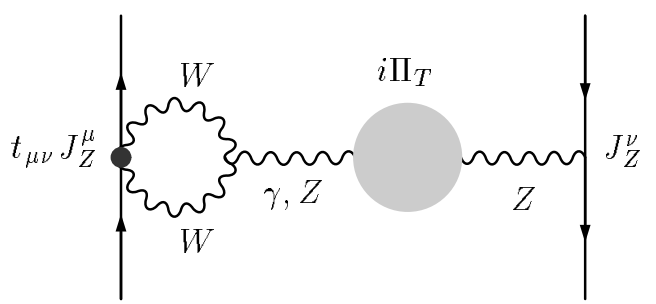

(c)

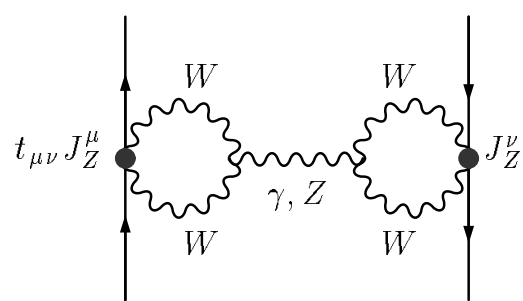

(d)

Figure 1 